\title{
Multiple Transmission Optimization of Medical Images in Recourse-Constraint Mobile Telemedicine Systems
}

\author{
Nan Jiang ${ }^{\mathrm{a}}$, Yi Zhuang ${ }^{\mathrm{b} *}$ and Dickson K.W. Chiu ${ }^{\mathrm{c}}$ \\ ${ }^{a}$ Hangzhou First People's Hospital, Hangzhou, P.R.China \\ ${ }^{\mathrm{b}}$ College of Computer \& Information Engineering, Zhejiang Gongshang University, Hangzhou, P.R.China \\ ${ }^{c}$ Faculty of Education, The University of Hong Kong, HKSAR, P.R.China
}

\begin{abstract}
Background and Objective: In the state-of-the-art image transmission methods, multiple large medical images are usually transmitted one by one which is very inefficient. The objective of our study is to devise an effective and efficient multiple transmission optimization scheme for medical images called MTO via analyzing the visual content of the multiple images based on the characteristics of a recourse-constraint mobile telemedicine system (MTS) and the medical images;

Methods: To better facilitate the efficient MTO processing, two enabling techniques, i.e., 1) NIB grouping scheme, and 2) adaptive RIB replicas selection are developed. Given a set of transmission images $(\Omega)$, the correlation of these transmission images is first explored, the pixel resolutions of the corresponding MIBs keep high, the NIBs are grouped into $k$ clusters based on the visual similarity in which the $k$ RIBs are obtained. An optimal pixel resolution for the RIBs is derived based on the current network bandwidth and their corresponding areas, etc. Then, the candidate MIBs and the $k$ RIBs are transmitted to the receiver node based on their transmission priorities. Finally, the IBs are reconstructed and displayed at the receiver node level for different users.

Results: The experimental results show that our approach is about $45 \%$ more efficient than the state-of-the-art methods, significantly minimizing the response time by decreasing the network communication cost while improving the transmission throughput;

Conclusions: Our proposed MTO method can be seamlessly applied in a recourse-constraint MTS environment in which the high transmission efficiency and the acceptable image quality can be guaranteed.
\end{abstract}

Keywords: Medical image, multi-resolution, mobile telemedicine system, batch transmission

Corresponding author: Yi Zhuang $(*)$

Tel: +86-571-87247080

Fax: +86-571-87247080

E-mail: zhuang@mail.zjgsu.edu.cn

Address: Apart.502, Unit 2, Blg.7, Qinchunfang, Hangzhou, P.R.China 


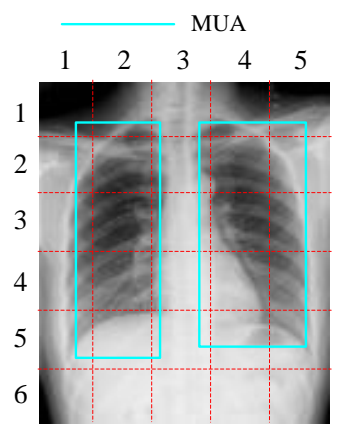

(a)

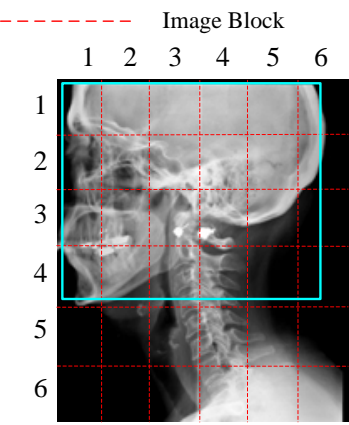

(b)

Fig. 1. Two different medical images with MUAs

\section{Introduction}

With the explosive growth of the number of medical images and the popularity of location-based service (LBS), locationbased medical image retrieval and browsing has been paid much attention to recently in mobile telemedicine systems (MTS) [1]. Telemedicine is the use of telecommunication and information technologies to provide clinical health care at a distance. It helps eliminate distance barriers and can improve access to medical services that would often not be consistently available in distant rural communities. Although medical image retrieval has been extensively studied, the state-of-the-art methods mainly focus on a single PC [2] or a traditional network environment [3]. A mobile telemedicine system provides us with a type of mobile and flexible computing infrastructure in which different users (e.g., physicians) in different places can obtain their desired information from the MTS conveniently. For mobile retrieval of large medical images, however, the network transmission cost will in large determine the total response time, especially in the rural or remote areas since the 4G mobile network services in these areas have not been provided. So the reduction of the transmission cost is very critical to the retrieval performance improvement. To achieve this, a scalable, high-throughput, location-based transmission scheme is generally required.

Although considerable amount of research efforts have been carried out on image transmission [4], most of them focus on two ways: 1) design of transmission protocol [5-9]; 2) image data compression [10-22]. The data transmission efficiencies of the two ways above are unsatisfactory because the response time is linearly increasing with the size of the transferred file. Moreover, for the transmission of several images, they are usually transferred one by one. There is no existing study on the multiple image transmission performance improvement from the perspective of the image batching, especially in the MTS environment. So to further speed up the transmission processing and improve user experiences, the paper proposes a Multiple Transmission Optimization method for large medical images ${ }^{1}$ in the $M T S$ environment, called the MTO, by exploring the content correlation of the transmission images.

\footnotetext{
${ }^{1}$ By default, the medical images in this paper refer to the grayscale image.
} 
The motivations of the MTO scheme are based on the following three key observations:

- Compared with other images, the backgrounds of medical images are relatively simple yet similar, which motivates us to identify and extract the medically useful areas (MUA) in medical images conveniently. The MUAs in Fig.1 are highlighted by the blue solid lines. Fig. 1 shows two illustrative medical images (a) and (b), which are equally partitioned into some blocks called image block(IB) by the red dash lines. For image (a), the color of the IB $a_{61}$ is visually similar to that of $b_{63}, b_{66}$ and $b_{55}$. In addition, $a_{62}, a_{63}$, and $a_{64}$ are similar to $b_{65}$ based on visual similarity computation. Meanwhile, $b_{51}, b_{52}, b_{56}, b_{61}$ and $b_{62}$ are also visually similar with each other. It is obvious that the total transmission overhead can be significantly reduced if the visually similar IBs can be represented by a representative IB(RIB) that is transmitted only once.

- Since any medical image includes some MUAs, the total image data size can be effectively reduced if the pixel resolution of the non-MUA part is moderately adjusted so that image examining can not be greatly affected. Thus, the total transmission cost is expected to be significantly decreased accordingly.

- The MTS is an image transmission-intensive application in which the mobile wireless network (MWN) bandwidth is usually unstable and limited, especially in the remote or rural areas. The transmission efficiency is very low. In this case, to significantly improve the transmission performance, it is urgent to devise a multi-transmission optimization approach. Moreover, it is acceptable for users (e.g., physicians) to improve the image transmission performance by moderately sacrificing the image quality (e.g., minor image distortion, etc).

The basic idea behind the MTO scheme works as follows. In the preprocessing step, for each image $I_{i}$, its corresponding MUAs are automatically identified and extracted by the discriminately trained deformable part model-based approach [27]. The images are then equally partitioned into some IBs that are placed in a slave node as replicas with different pixel resolutions and transmission priorities. As the IBs for the non-MUA parts of the images, denoted as NIB, are critically important to the performance improvement of the multiple image transmission optimization in which they can be approximately represented by their corresponding RIBs, their pixel resolutions are adjusted moderately based on the network bandwidth and their areas, etc. Their corresponding transmission priorities are lower than the IBs of the MUAs (called MIB) such that the main part (i.e., MUA) of the image can be transmitted and displayed in priority. Once the pre-processing step is completed, the next step is to perform the multiple transmission optimization processing for medical images. Given a set of transmission images $(\Omega)$, the correlation of these transmission images is first explored, the pixel resolutions of the corresponding MIBs keep high, the NIBs are grouped into $k$ clusters by AP-clustering algorithm [29] based on the visual similarity in which the $k$ RIBs are obtained. An optimal pixel resolution for the RIBs is derived based on 
the current network bandwidth and their corresponding areas, etc. Then, the candidate MIBs and the $k$ RIBs are transmitted to the receiver node based on their transmission priorities. Finally, the IBs are reconstructed and displayed at the receiver node level for different users.

The challenges of designing the high performance multi-transmission optimization processing of medical images include the four main aspects: 1) How to group the medical images together: since for the NIBs from the transmission images, there exist some visually similar NIBs, which motivate us to cluster the similar NIBs together; 2) High computation cost in medical image transmission: most of medical images are characterized by high pixel resolution, high-dimensional, and large-scale. So, the transmission costs of such medical images are very high; 3) Resource-Constraint MWN: the power capacities of the mobile devices are very limited. The display resolutions of such mobile devices are often low. Furthermore, the bandwidth in the $M W N$ is limited, how to transmit such a batch of large images simultaneously in the resourceconstraint MWN is challenging; 4) Instability and heterogeneity of the MTS: the nodes in the MWN are instable, that means, some nodes may be down or connected intermittently to the network. Further, the bandwidth between any two nodes may vary with time. There is no guarantee that the total response time of each transmission will be similar. To address the above challenges, an efficient multi-transmission optimization scheme for medical images (MTO) in the recourse-constraint MTS environment is proposed. To the best of our knowledge, this is the first study on the multiple transmission optimization for medical images.

This paper is structured in the followings. Section 2 provides the background of the related techniques. Section 3 entails methodologies that are employed in this study as well as the MTO method. After that, Section 4 puts forward the experimental results and discussions whereas the conclusions are summarized in Section 5.

\section{Background}

In this section, the paper reviews some background of image data transmission techniques that have been extensively studied for several decades [4]. The state-of-the-art methods can be mainly divided into two categories: 1) transmission protocol design [5 - 9]; and 2) image data encoding and compression [10 -22].

In the first category, Turner and Peterson [5] first proposed a wireless image data transmission method from end to end. Danskin et al. [6] presented a fast lossy Internet image transmission scheme(FLIT) for compressed images which eliminates retransmission delays by strategically shielding important portions of the image with redundancy bits. Compared with the traditional TCP protocol, Raman et al. [7] designed a lossy image transmission protocol (ITP) which is more suitable for image data transmission. Due to the high packet error rates and the need for retransmission, recently Aziz et al. [9] have designed a novel architecture and protocol for energy efficient image processing and communication over wireless 
sensor networks.

In the second category, Kim and Song [10] presented a pyramid-structured progressive image transmission method using quantization error delivery in transform domains. Chang et al. [11] proposed a new scheme of progressive image transmission (PIT) based on bit-plane method(BPM), which transmits the most significant portion of a picture, followed by less important parts. Hashimoto et al. [12] presented a hierarchical image transmission system for telemedicine using segmented wavelet transform and Golomb-Rice codes. Ruiz et al. [13] designed an image compression algorithm to support progressive image transmission. To achieve a faster transmission performance, Chang et al. [14] improved the BPM method by color guessing called the guessing by neighbors method which uses interleaved pixels for transmission. Chang and Wu [15] proposed a color image progressive transmission method by common bit map block truncation coding approach. Lin et al. [16] presented a compound image compression algorithm for real-time applications of computer screen image transmission. Sun et al. [17] considered a progressive image transmission system over wireless channels by combining joint source-channel coding, space-time coding, and orthogonal frequency division multiplexing. Chang et al. [18] design a strategic decomposition scheme for adaptive image transmission processing. Gao et al. [19] proposed a robust image transmission scheme for wireless channels based on compressive sensing. Boluk et al. [20] designed a image transmission over wireless sensor networks. Arslan et al. [21] proposed a generalized unequal error protection LT codes for progressive data transmission. Xua et al. [22] proposed an adaptive FEC coding and cooperative relayed wireless image transmission.

Besides the above two categories, Maani et al. [23] designed a parallel method to improve medical image transmission processing. Recently, Hemalatha et al. [24] designed an energy-efficient image transmission in wireless multimedia sensor networks using block-based Compressive Sensing. Manimurugan et al. [25] and Ai-Hai et al. [26] introduced several crypto-based algorithms for secure medical image transmission, respectively.

In our previous work, Zhuang et al. [3] have explored the feasibility of content-aware and multi-resolution-based medical image transmission scheme in which only two factors, namely, the image content and the network bandwidth are considered to optimize the transmission processing. Based on the above transmission model, Zhuang et al. [28] designed a personalized social image transmission scheme in mobile wireless network.

Different from the above state-of-the-art methods, the paper proposes a multiple transmission optimization scheme for medical images via the analysis of the image contents in which a set of the transmission images are transmitted to receiver node in a batch manner. To the best of our knowledge, this is the first attempt to improve the image transmission performance from the perspective of the multiple transmission optimization. 


\section{Methodology}

\subsection{Preliminaries and problem definition}

First of all, the main symbol notations are summarized in Table 1.

Table 1 . Frequently used symbols

\begin{tabular}{|l|l|}
\hline Symbol & Description \\
\hline$\Omega$ & a set of transmission images \\
\hline$I_{i}$ & the $i$-th transmission image and $I_{i} \in 2$ \\
\hline$\Psi$ & a NIB set for the transmission images \\
\hline$U$ & a set of submission users \\
\hline$I B_{i}$ & the $i$-th image block \\
\hline$M I B_{i j}$ & the $j$-th image block of the MUAs in the $i$-th image \\
\hline$N I B_{i j}$ & the $j$-th image block of the non-MUA part in the $i$-th image \\
\hline$I B G_{k}$ & the $k$-th image block group \\
\hline$R I B(k)$ & the representative image block in the $k$-th $I B G$ \\
\hline$|\bullet|$ & the number of $\bullet$ \\
\hline$M U A_{j}$ & the $j$-th MUA in a medical image and $j \in[1,|M U A|]$ \\
\hline$U_{i}$ & the $i$-th user and $i \in[1,|U|]$ \\
\hline$N$ & the non-MUA part of the image \\
\hline$D_{L}, D_{U}$ & the lower and upper bounds of the dots per inch, respectively \\
\hline$E_{\mathrm{L}}, E_{\mathrm{U}}$ & the lower and upper bounds of the bandwidth, respectively \\
\hline$\alpha_{i}$ & the number of MIBs in the $i$-th image \\
\hline$\beta_{i}$ & the number of RIBs in the $i$-th image \\
\hline$\Delta_{i n i}$ & initial granularity value for the pixel resolution \\
\hline$\Delta_{o p t}$ & optimal granularity value for the pixel resolution \\
\hline$\delta$ & granularity value for the size of image blocking \\
\hline
\end{tabular}

DEFINITION 1. A mobile wireless network (MWN) is a graph which is represented by a triplet:

$$
M W N=<N, E, T>
$$

where $N$ refers to a set of nodes, E means a set of edges representing the network bandwidths for transmission at time $T$.

In the above definition, as shown in Fig. 2, due to the instability and heterogeneity of the MWN environment, the bandwidth of any two nodes in $M W N$ may be different and variant with the change of the time. In addition, the data transmission distance in the mobile wireless network is limited.

DEFINITION 2. The nodes in the MWN can be logically divided into three categories: the sender node $\left(N_{S}\right)$, the slave node $\left(N_{L}\right)$, and the receiver node $\left(N_{R}\right)$, formally denoted as $N=N_{S} \cup N_{L} \cup N_{R}$, where

- $\quad N_{S}$ is responsible for collecting and analyzing the receivers' transmission requests and the current network bandwidth to obtain an optimal image block transmission pixel resolution; 


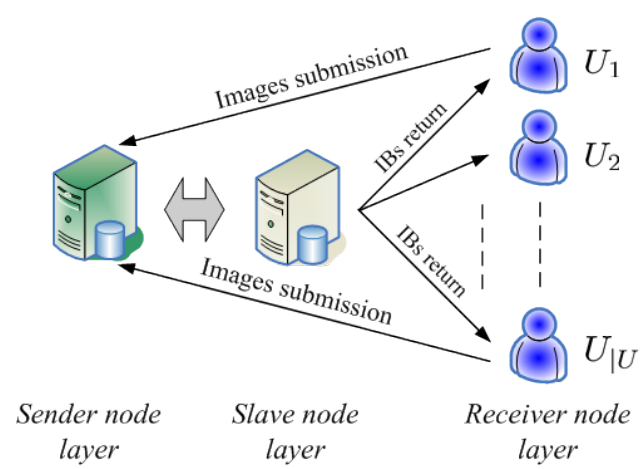

Fig. 2. The three layer architecture of a MWN

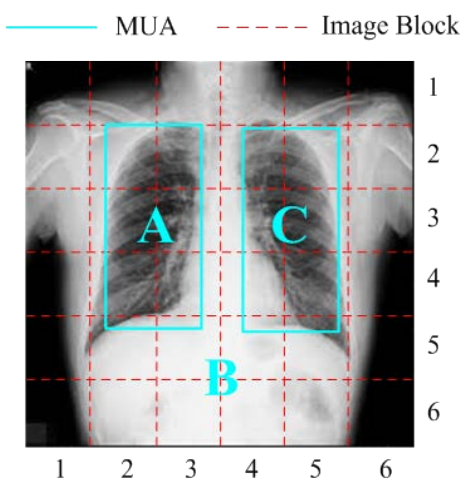

Fig. 3. Two $\operatorname{MUAs}(A$ and $C)$ in a medical image $(\delta=6 \times 6)$

- $N_{L}$ is responsible for: 1) storing the IB replicas with different pixel resolutions and transmission priorities, and 2) sending the images to the receivers;

- $N_{R}$ is responsible for: 1) images submission; 2) receiving, reconstructing, and displaying the images for different receivers.

As mentioned in Section 1, for each image, in most cases, there exist some salient objects that users are interested in. The regions of such salient objects (i.e. organs) are called medically useful area (MUA) which can be preliminarily detected by the discriminately trained deformable part model-based approach [27].

DEFINITION 3. A medically useful area (MUA) in an image can be modeled by a five-tuple:

$$
M U A_{i}=<i, S, \text { pos, } d p i, T P>
$$

where $i$ is the ID number of the MUA, $S$ is the area value of the MUA, pos refers to the position of the MUA in an image, dpi refers to the dots per inch of the MUA, TP is transmission priority of the MUA.

DEFINITION 4. A non-MUA part of an image, denoted as N, can be modeled by a two-tuple:

$$
N=<S, d p i>
$$

where $S$ is the area value of the $N$; dpi refers to the dots per inch of the $N$;

DEFINITION 5 (IMAGE BLOCK, IB). An image block can be modeled as a six-tuple:

$$
I B=<\text { bid, pos, dpi, TP, IBGID, uID > }
$$

where bid refers to the block ID, pos is the coordinate of the block in the image, dpi is the dots per inch of the block, TP is the transmission priority of the block, IBGID is the image block group(IBG) ID where the IB belongs to, and uID means the user ID by which the transmission image submits to.

Based on Definitions 3, 4 and 5, Fig. 3 illustrates that there are two MUAs ( $A$ and $C$ ) and one $N$ (i.e., $B$ ) in the image in 
which the IBs have been drawn by red dash lines and the granularity $\delta$ equals to $6 \times 6$.

The problem can be defined as follows: given $|U|$ user image transmission requests at time $T$, a MTO processing of the $|U|$ images is to simultaneously transmit the images to the receivers with the minimal communication, I/O and CPU costs via uniformly analyzing the visual contents of the images and the network bandwidth. The goal of our proposed MTO method is to get a tradeoff between the transmission efficiency in the MTS environment and the acceptable image quality.

\subsection{Enabling Techniques}

In this section, to better facilitate the multiple medical images transmission processing, two enabling techniques are introduced: 1) NIB grouping scheme, and 2) adaptive RIB replicas selection.

\subsubsection{NIB grouping scheme}

Given a set of transmission images $(\Omega)$, the first step of the MTO processing is to analyze the correlated IBs from the nonMUA regions in the images from $\Omega$ by exploring the visual similarity of them.

\section{- Blocking the Images}

In this preprocessing step, as described in Definition 5, a medical image is first equally partitioned into some IBs among which there are two kinds of IBs (i.e., MIB and NIB) in terms of their positions in the image.

DEFINITION 6 (MIB). A MIB is an image block that intersects with a MUA or is contained by a MUA, formally denoted as:

$$
M I B=\left\{I B_{i j} \mid I B_{i j} \cap M U A_{k} \neq \emptyset\right\}
$$

where $i$ is a row ID and $i \in[1, \alpha], j$ is a column ID and $j \in[1, \beta]$, and $k \in[1,|M U A|]$.

DEFINITION 7 (NIB). A NIB is an image block which is contained by the non-MUA part (N) of an image, formally represented by:

$$
N I B=\left\{I B_{i j} \mid I B_{i j} \cap N=I B_{i j}\right\}
$$

where the definitions of $i$ and $j$ are same as that in Definition 6.

Based on the above Definitions (6-7), the $M I B s$ in Fig. 3 are $I B_{22}, I B_{23}, I B_{24}, I B_{25}, I B_{32}, I B_{33}, I B_{34}, I B_{35}, I B_{42}, I B_{43}, I B_{44}$, $I B_{45}, I B_{52}, I B_{53}, I B_{54}$ and $I B_{55}$. The rest of the $I B$ s are $N I B s$.

\section{- Grouping the NIBs}

For the RIBs extracted from the transmission images $(\Omega)$, their corresponding similarity matrix is first calculated based on visual similarity distance which is defined in Definition 8.

DEFINITION 8 (SIMILARITY MATRIX). Given an NIB set $(\Psi)$, its corresponding similarity matrix(SM) is a matrix in which 
each element $\left(d_{i j}\right)$ is the visual similarity (i.e., Euclidean distance) of the two NIBs (i.e., $N_{I B}$ and $\left.N I B_{j}\right)$, formally denoted as:

$$
S M(\Psi)=\left[\begin{array}{cccc}
d_{11} & d_{12} & \cdots & d_{1|\Psi|} \\
d_{21} & d_{22} & \cdots & d_{2|\Psi|} \\
\vdots & \vdots & \vdots & \vdots \\
d_{|\Psi| 1} & d_{|\Psi| 2} & \cdots & d_{|\Psi||\Psi|}
\end{array}\right]
$$

where $N I B_{i}, N I B_{j} \in \Psi$, and $i, j \in[1,|\Psi|]$.

For the NIBs of the transmission images, the $k I B G s$ are obtained by using the AP-cluster [29] in which the NIBs are visually similar. Algorithm 1 shows the NIB grouping processing for obtaining the $k I B G$ s. For the NIBs in each $I B G$, a cluster center NIB is denoted as a representative NIB called RIB in this IBG. The RIB can approximately represent all of the NIBs in the corresponding IBG. The computational complexity is $O\left(|\Psi|^{2}\right)$.

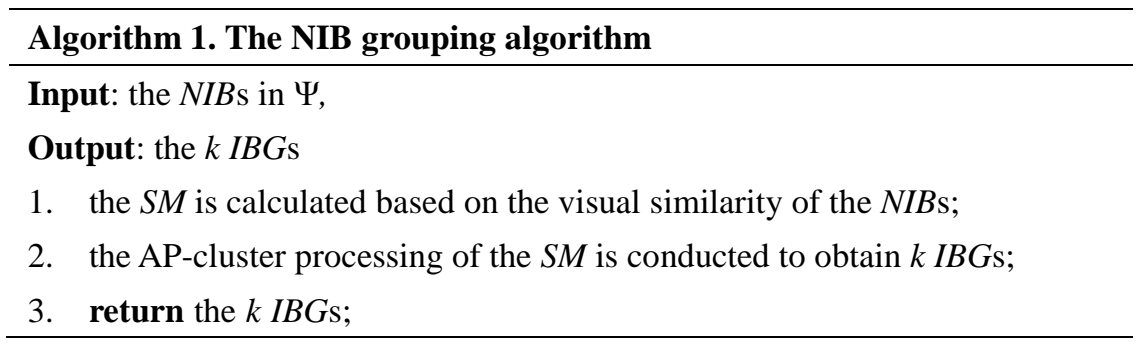

\subsubsection{Adaptive RIB replicas selection}

As mentioned before, since different pixel resolutions corresponds to different data sizes of a medical image. The costs to transmit an unprocessed medical image with such a big size to the receiver nodes is usually very high, especially in a MWN environment since the network bandwidth is limited and unstable. Based on the above analysis, the subsection proposes an

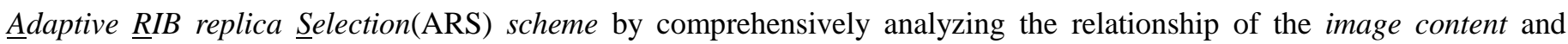
network bandwidth.

\section{- Choosing an Optimal Pixel Resolution}

The basic idea of the ARS method is that for a same medical image, the image with different pixel resolutions can be transferred according to the variance of the network bandwidth. Specifically, with a high network bandwidth, a highresolution image replica is transferred in a reasonable short period of time $\left(\theta_{T}\right)$. On the contrary, in order to get a shorter response time, a lower-resolution version can be sent to the receiver node with a lower network bandwidth. Although reducing the pixel resolution of the whole image can reduce the transmission cost, some salient objects (i.e., MUA), however, cannot be clearly examined by physicians and may possibly lead to the misdiagnosis. Therefore, compared with the resolution of the non-MUA part of the image, the MUAs need to be presented with a higher pixel resolution.

Based on this consideration, the objective of the ARS method is to get a tradeoff between the image quality, the transmission cost under different resolutions and available network bandwidths with a constraint of the time threshold $\left(\theta_{T}\right)$. 
Specifically, let us first denote the average resolution of the images in $\Omega$ as $d p i \in\left[D_{L}, D_{U}\right]$, where $D_{\mathrm{L}}$ and $D_{\mathrm{U}}$ denote the lower and upper bounds of the dots per inch (dpi) for images in $\Omega$, respectively. Suppose that $|\Omega|$ images are prepared for transmission, there are $\sum_{i=1}^{|\Omega|} \alpha_{i}$ MIBs and $\sum_{i=1}^{|\Omega|} \beta_{i}$ NIBs that are grouped into $k$ clusters in which $k$ RIBs are obtained. So, the $\sum_{i=1}^{|\Omega|} \alpha_{i}$ MIBs and $k$ RIBs are prepared to be transmitted. The total area of the IBs (i.e., MIBs and RIBs) and their corresponding pixel resolutions are met in Eq.(8):

$$
\sum_{i=1}^{|\Omega|} \sum_{j=1}^{\alpha_{i}}\left(M I B_{i j} . S \cdot M I B_{i j} \cdot d p i^{2}\right)+\sum_{j=1}^{k}\left(R I B_{j} . S \cdot R I B_{j} \cdot d p i^{2}\right)=\left(\sum_{i=1}^{|\Omega|} \sum_{j=1}^{\alpha_{i}} M I B_{i j} \cdot S+\sum_{j=1}^{k} R I B_{j} \cdot S\right) \cdot d p i^{2}
$$

Solving Eq.(8), the average dpi of the transmission images (i.e., images in $\Omega$ ) can be derived in Eq.(9):

$$
d p i=\sqrt{\frac{\sum_{i=1}^{|\Omega|} \sum_{j=1}^{\alpha_{i}}\left(M I B_{i j} \cdot S \cdot M I B_{i j} \cdot d p i^{2}\right)+\sum_{j=1}^{k}\left(R I B_{j} \cdot S \cdot R I B_{j} \cdot d p i^{2}\right)}{\sum_{i=1}^{|\Omega|} \sum_{j=1}^{\alpha_{i}}\left(M I B_{i j} \cdot S\right)+\sum_{j=1}^{k}\left(R I B_{j} \cdot S\right)}}
$$

In addition, the bandwidth of the $j$-th edge is defined as: $E_{C} \in\left[E_{\mathrm{L}}, E_{\mathrm{U}}\right]$, where $E_{\mathrm{L}}$ and $E_{\mathrm{U}}$ are the lower and upper bounds of the bandwidth of the $j$-th edge, respectively. Note that, the above bandwidth is a theoretical value larger than the actual one. For the current network bandwidth $E_{C}$, Eq. (10) can be met:

$$
E_{c} \in\left[E_{\mathrm{L}}+\frac{(i-1) \cdot\left(E_{\mathrm{U}}-E_{\mathrm{L}}\right)}{\Delta}, E_{\mathrm{L}}+\frac{i \cdot\left(E_{\mathrm{U}}-E_{\mathrm{L}}\right)}{\Delta}\right]
$$

Since $i$ is an integer, so

$$
i=\left\lceil\frac{\left(E_{c}-E_{\mathrm{L}}\right) \cdot \Delta}{E_{\mathrm{U}}-E_{\mathrm{L}}}+1\right\rceil
$$

where $\lceil\bullet\rceil$ refers to the integral part of $\bullet$.

Based on the assumption that in most cases, the average $d p i$ of the transmission images $(\Omega)$ is proportional to the network bandwidth $\left(E_{C}\right)$, so their corresponding $d p i$ in $\Omega$ under $E_{C}$ can be derived as follows:

$$
\begin{gathered}
d p i=D_{L}+\frac{i \cdot\left(D_{u}-D_{L}\right)}{\Delta} \\
d p i=D_{L}+\left\lceil\frac{\left(E_{c}-E_{\mathrm{L}}\right) \cdot \Delta}{E_{\mathrm{U}}-E_{\mathrm{L}}}+1\right\rceil \cdot \frac{D_{\mathrm{U}}-D_{\mathrm{L}}}{\Delta}
\end{gathered}
$$

where $i \in[1, \Delta]$.

In Eq.(13), the whole image is regarded as an object to be processed. The pixel resolution of the whole image can be adjusted according to the variance of the network bandwidth. This method, however, may reduce the pixel resolution of the MUAs so much that the user cannot clearly examine the image. Therefore, in the preprocessing step, as shown in Fig. 4(a), the MUAs in the image are firstly identified by two blue dash rectangles manually, namely $\mathrm{MUA}_{1}$ and MUA 2 . Fig. 4(b) shows that the resolutions of the two MUAs in Figs. 4(a) and 4(b) are likely unchanged, and the resolution of the non-MUA $\operatorname{part}(N)$ in Fig. 4(b), however, is decreased dramatically.

Combing Eqs.(8) and (13), Eq.(14) can be derived: 


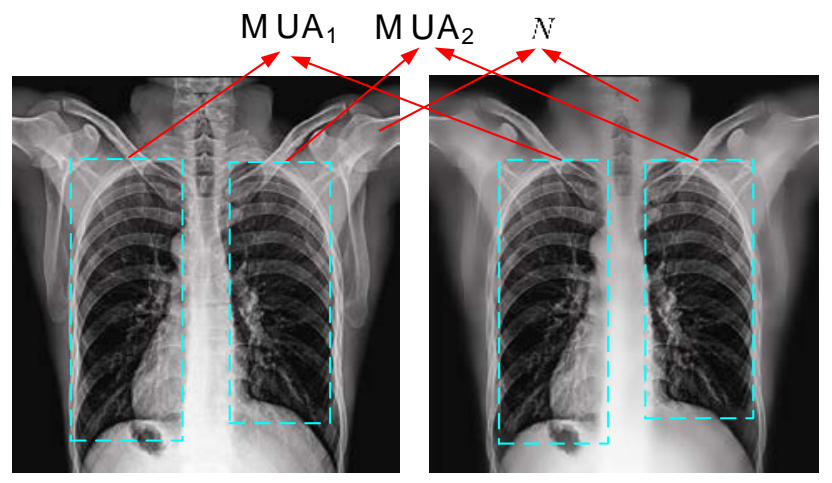

(a). Before

(b). after

Fig. 4. Comparison of the pixel resolutions of a same image

$$
\sqrt{\frac{\sum_{i=1}^{|\Omega|} \sum_{j=1}^{\alpha_{i}}\left(M I B_{i j} \cdot S \cdot M I B_{i j} \cdot d p i^{2}\right)+\sum_{j=1}^{k}\left(R I B_{j} \cdot S \cdot R I B_{j} \cdot d p i^{2}\right)}{\sum_{i=1}^{|\Omega|} \sum_{j=1}^{\alpha_{i}}\left(M I B_{i j} \cdot S\right)+\sum_{j=1}^{k}\left(R I B_{j} \cdot S\right)}}=D_{L}+\left\lceil\frac{\left(E_{c}-E_{\mathrm{L}}\right) \cdot \Delta}{E_{\mathrm{U}}-E_{\mathrm{L}}}+1\right\rceil \cdot \frac{D_{\mathrm{U}}-D_{\mathrm{L}}}{\Delta}
$$

Solving Eq.(14), the dpi of the RIBs from the non-MUA part in the images $(\Omega)$ can be derived as:

$$
R I B . d p i=\sqrt{\frac{\left(\sum_{i=1}^{|\Omega|} \sum_{j=1}^{\alpha_{i}} M I B_{i j} . S+\sum_{j=1}^{k} R I B_{j} . S\right) \cdot\left(D_{L}+\left[\frac{\left(E_{c}-E_{\mathrm{L}}\right) \cdot \Delta}{E_{\mathrm{U}}-E_{\mathrm{L}}}+1\right] \cdot \frac{D_{\mathrm{U}}-D_{\mathrm{L}}}{\Delta}\right)^{2}-\sum_{i=1}^{|\Omega|} \sum_{j=1}^{\alpha_{i}}\left(M I B_{i j} . S \cdot M I B_{i j} \cdot d p i^{2}\right)}{\sum_{j=1}^{k} R I B_{j} . S}}
$$

Based on the above analysis, if $\Delta$ is fixed, with increase of network bandwidth, the optimal transmission pixel resolution is increasing accordingly. Meanwhile, if the bandwidth is fixed, when $\Delta$ increases, the optimal transmission pixel resolution will decrease accordingly.

\section{- Modeling $\Delta$ and $\underline{E}_{\underline{C}}$}

Since the larger $\Delta$ is, the larger the storage cost of the IB replicas is. To minimize the above total storage cost, $\Delta$ needs to be minimized. To obtain an optimal $\Delta$, suppose that the image transmission processing can be finished in a transmission deadline $\left(\theta_{T}\right)$ set by user, so:

$$
T_{T}=T_{0}+\frac{\operatorname{Size}(\Omega)}{\operatorname{BWidth}\left(E_{c}\right)} \leq \theta_{T}
$$

where

— Size $(\Omega)$ is the data size of the jĐj transmission images, represented as:

$$
\operatorname{Size}(\Omega)=\left(\sum_{i=1}^{|\Omega|} \sum_{j=1}^{\alpha_{i}}\left(M I B_{i j} \cdot S \cdot M I B_{i j} \cdot d p i^{2}\right)+\sum_{j=1}^{k}\left(R I B_{j} \cdot S \cdot R I B_{j} \cdot d p i^{2}\right)\right) \cdot B i t \cdot C R
$$

where Bit means color bit, and Bit can be 8,16 , or $24, C R$ is an image compression ratio and $C R \in[0,1]$;

- $T_{0}$ is the start-up transmission time;

- $B$ Width $\left(E_{C}\right)$ is the actual network bandwidth, denoted as $B W i d t h\left(E_{C}\right)=E_{C} \cdot T R$, where $E_{C}$ is the current network bandwidth, $T R$ means the attenuation rate of the bandwidth and $T R \notin 0,1]$. 
Eq. (16) can be rewritten as follows:

$$
\sum_{i=1}^{|\Omega|} \sum_{j=1}^{\alpha_{i}}\left(M I B_{i j} \cdot S \cdot M I B_{i j} \cdot d p i^{2}\right)+\sum_{j=1}^{k}\left(R I B_{j} . S \cdot R I B_{j} \cdot d p i^{2}\right) \leq \frac{\left(\theta_{T}-T_{0}\right) \cdot E_{c} \cdot T R}{B i t \cdot C R}
$$

Meanwhile, based on Eqs.(8) and (16), Eq.(18) can be derived:

$$
\left(\sum_{i=1}^{|\Omega|} \sum_{j=1}^{\alpha_{i}} M I B_{i j} \cdot S+\sum_{j=1}^{k} R I B_{j} \cdot S\right) \cdot d p i^{2} \leq \frac{\left(\theta_{T}-T_{0}\right) \cdot E_{c} \cdot T R}{B i t \cdot C R}
$$

Combing Eqs.(13) and (18), Eq.(19) can be obtained:

$$
D_{L}+\left\lceil\frac{\left(E_{c}-E_{\mathrm{L}}\right) \cdot \Delta}{E_{\mathrm{U}}-E_{\mathrm{L}}}+1\right\rceil \cdot \frac{D_{\mathrm{U}}-D_{\mathrm{L}}}{\Delta} \leq \sqrt{\frac{\left(\theta_{T}-T_{0}\right) \cdot E_{c} \cdot T R}{\operatorname{Bit} \cdot C R \cdot\left(\sum_{i=1}^{|\Omega|} \sum_{j=1}^{\alpha_{i}} M I B_{i j} \cdot S+\sum_{j=1}^{k} R I B_{j} \cdot S\right)}}
$$

Then, $\Delta$ can be approximately solved by Eq.(19).

$$
\Delta \geq \frac{D_{U}-D_{L}}{\sqrt{\frac{\left(\theta_{T}-T_{0}\right) \cdot E_{c} \cdot T R}{B i t \cdot C R \cdot\left(\sum_{i=1}^{|\Omega|} \sum_{j=1}^{\alpha_{i}} M I B_{i j} \cdot S+\sum_{j=1}^{k} R I B_{j} \cdot S\right)}}-D_{L}-\frac{\left(E_{c}-E_{L}\right) \cdot\left(D_{U}-D_{L}\right)}{E_{U}-E_{L}}}
$$

To minimize the total storage cost of the IB replicas, the value of $\Delta$ should be minimized too. Solving Eq.(20), $\Delta$ can be approximately represented below:

$$
\Delta \approx\left\lceil\frac{D_{U}-D_{L}}{\sqrt{\frac{\left(\theta_{0}-T_{0}\right) \cdot E_{c} \cdot T R}{B i t \cdot C R \cdot\left(\sum_{i=1}^{|\Omega|} \sum_{j=1}^{\alpha_{i}} M I B_{i j} \cdot S+\sum_{j=1}^{k} R I B_{j} \cdot S\right)}}-D_{L}-\frac{\left(E_{c}-E_{L}\right) \cdot\left(D_{U}-D_{L}\right)}{E_{U}-E_{L}}}\right\rceil
$$

For example, given a set of transmission images $(\Omega)$, assume that the total areas of the corresponding MIBs and the $k$ RIBs are 20 inch $^{2}$ and 5 inch $^{2}$, respectively. The minimal and maximal pixel resolutions of an image are: $D_{\mathrm{L}}=20$ and $D_{\mathrm{U}}=$ 100 , respectively. The bandwidth of the wireless network ranges from $10 \mathrm{MB} / \mathrm{Sec}$. to $100 \mathrm{MB} / \mathrm{Sec}$., namely, $E_{\mathrm{L}}=10 \mathrm{MB} / \mathrm{Sec}$., $E_{\mathrm{U}}=100 \mathrm{MB} /$ Sec. $T_{0}=0.1 \mathrm{~s}, \theta_{\mathrm{T}}=1 \mathrm{~s}, T R=0.1, C R=0.01$, Bit=8.

Based on Eq.(21), if the current network bandwidth $\left(E_{j}\right)$ is $50 \mathrm{MB} / \mathrm{Sec}$, then the optimal value of $\Delta$ is 7 .

\section{- Optimal ID of RIB Replica}

In the above, an optimal granularity( $\Delta)$ has been obtained. Next, how to choose an optimal ID of each $R I B$ replica among its corresponding $\Delta$ replicas is a critical issue to study.

For the non-MUA part of the image, as the lower and upper bounds of the $d p i$ (i.e., $D_{L}, D_{U}$ ), the ID number of the RIB replica (i) and $\Delta$ are met in Eq.(22).

$$
D_{L}+\frac{D_{U}-D_{L}}{\Delta} \cdot i=R I B . d p i
$$

where $\Delta$ is equal to that of in Eq.(21).

As $i$ is an integer, solving Eq.(22), the RIB replica ID can be approximately derived as:

$$
i=\left\lceil\frac{\left(R I B \cdot d p i-D_{L}\right) \cdot \Delta}{D_{U}-D_{L}}\right\rceil
$$




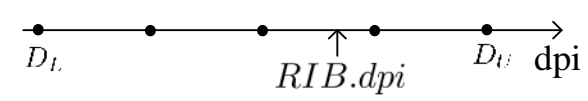

Fig. 5. The selection of optimal RIB replica ID $(\Delta=4)$

Fig. 5 illustrates an example of the selection of optimal $R I B$ replica where $\Delta=4$. To obtain an optimal $R I B$ replica ID, if $\left|D_{L}+\frac{D_{U}-D_{L}}{\Delta} \cdot i-R I B . d p i\right| \leq\left|D_{L}+\frac{D_{U}-D_{L}}{\Delta} \cdot(i+1)-R I B . d p i\right|$, then the optimal ID of the RIB replica is derived in Eq.(24):

$$
i_{o p t}=\left\lceil\frac{\left(R I B \cdot d p i-D_{L}\right) \cdot \Delta}{D_{U}-D_{L}}\right\rceil
$$

Otherwise, the optimal ID of the RIB replica is as follows:

$$
i_{o p t}=\left\lceil\frac{\left(R I B \cdot d p i-D_{L}\right) \cdot \Delta}{D_{U}-D_{L}}\right\rceil+1
$$

\subsection{The Мто algorithm}

With the support of the above enabling techniques, the MTо processing of the medical images can be efficiently transferred in the mobile wireless network environment. Before introducing the MTO algorithm, a pre-processing step is required. As mentioned in Section 3.1, in the preprocessing step, the MUAs of the all images are first identified [27] and stored in the database. Then, the images are equally partitioned into some IB (i.e., NIB and MIB) replicas in which the NIBs are stored based on different granularities and the MIBs are stored with their original pixel resolutions.

Generally speaking, in the state-of-the-art image data transmission schemes, a complete image is transferred as an object in which the transmission priority of each $I B$ in the image is equal. Thus, it is possible that the important MUAs in the image be displayed later than the non-MUA part. Moreover, for the medical images with high pixel resolutions, this transmission method, however, will lead to the increase of the failure in the transmission processing. Once the transmission failure is occurred, the image needs to be re-transmitted which results in a higher transmission cost. To overcome this technical bottleneck and support the robust transmission of the large image data, the transmission priority(TP) of each IB can be defined in Eq.(26).

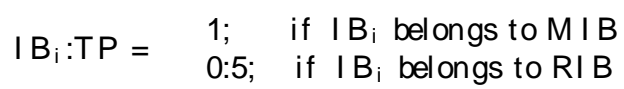

According to the different priorities of the IBs, they can be transferred in terms of the priority in a descending order, which not only ensures the robustness of data transmission but guarantees that the important information can be transferred in advance.

Algorithm 2 summarizes the detailed steps of our proposed Мто algorithm. As illustrated in Fig. 6, first of all, when a batch of image transmission requests $(\Omega)$ are submitted to the sender node level $N_{S}$ from the receiver one $N_{R}$, their 


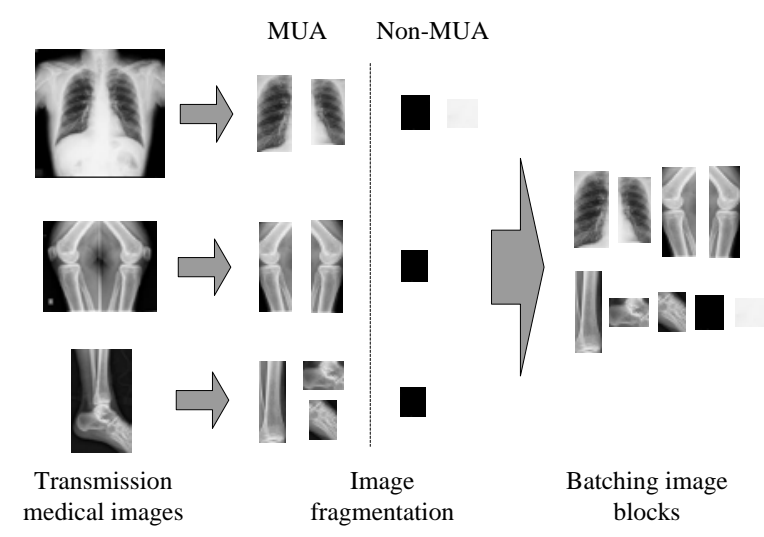

Fig. 6. The NIBs grouping processing

corresponding NIBs are first identified and extracted from the images in $\Omega$ (line 1). Then, the NIB grouping processing is performed to obtain the $k$ RIBs (line 2); after that, the network bandwidth and the corresponding areas of the IBs (i.e., MIBs and RIBs), etc., are collected and analyzed to obtain an optimal transmission pixel resolution for the RIB replicas (lines 3-7); in lines 6-8, the transmission priorities of each IB are assigned based on Eq.(26) and sorted; next, the IBs at the slave node can be sent to the receiver one based on their transmission priorities in a descending order (line 9); finally, in lines 10-11, once the IBs are arrived at the receiver node level, the IBs reconstruction processing is required. In this step, the MIBs are placed based on their positions (i.e., MIB.pos) and the corresponding user IDs (i.e., uID) in priority. The NIB replicas can be replaced by the corresponding RIB replicas and are placed based on their positions (i.e., NIB.pos) and the corresponding uIDs.

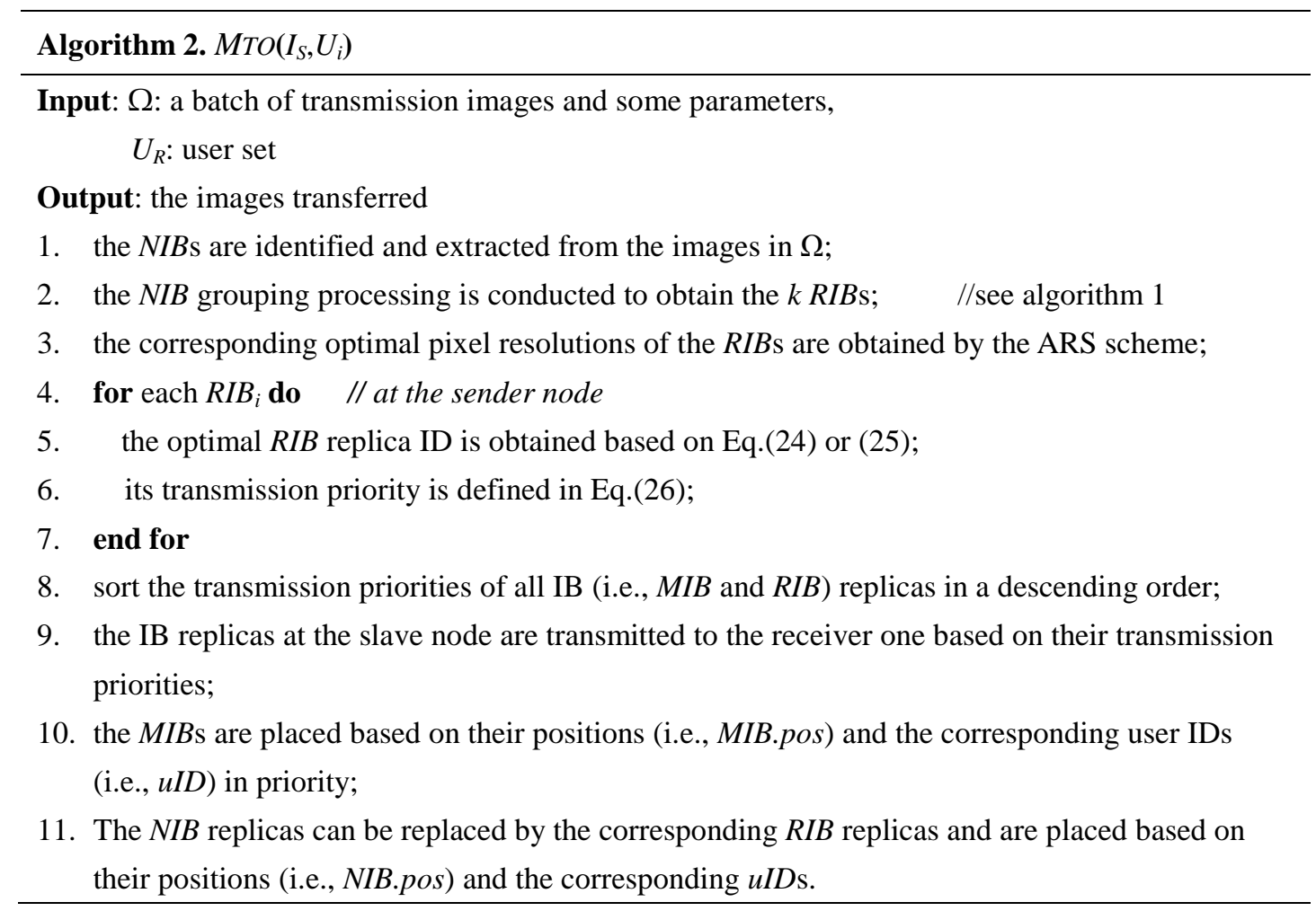




\section{Results and Discussion}

To verify the efficiency of the proposed MTO method, extensive simulation experiments are conducted to demonstrate the transmission performance.

The image receiver client has a Qualcomm ${ }^{\circledR}$ Snapdragon ${ }^{\mathrm{TM}} 600$ processor $1.7 \mathrm{GHz}$ quad-core $\mathrm{CPU}$, and a screen of 5.9 inch with full HD 1080p. The client system runs on the Android platform [30] and is implemented with the Java language. The sender node and the slave one are connected via 1Gbps network links. In the slave node, the IB replicas with different pixel resolutions are stored in a file system and some structured information is recorded by the MySQL [31]. Each node has a $2.7 \mathrm{GHz}$ quad-core Xeon processor, 2.0GB memory, and 1TB hard disk. The maximum data communication rate is 150 Mbps in the wireless network.

The medical image datasets adopted are from two ways: 1) real dataset: 100,000 medical images are downloaded from the medical image archive [32] in which the image data size ranges from 100k to 800k; 2) synthetic dataset: to evaluate the effect of data size on the image transmission performances, five groups of the medical image data have been synthesized in which the data size of each image are $1 \mathrm{MB}, 5 \mathrm{MB}, 10 \mathrm{MB}, 50 \mathrm{MB}$, and $100 \mathrm{MB}$, respectively. To objectively evaluate the Мто method, by default, each experiment runs 5 times to obtain the average values.

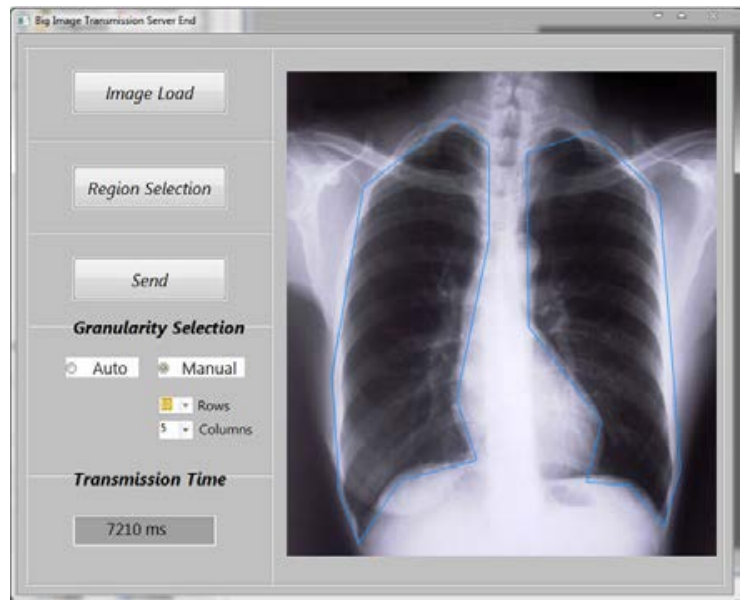

(a). A backend interface

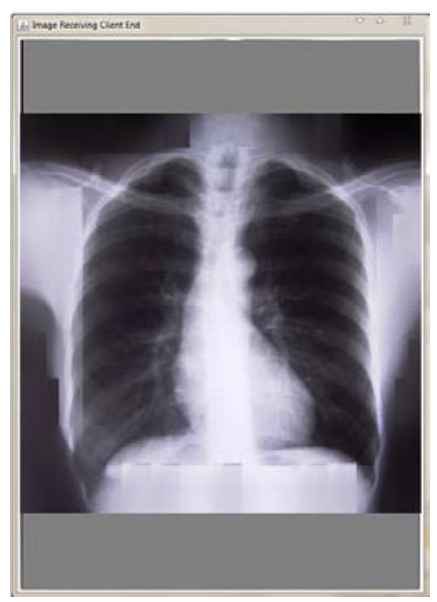

(b). A client end interface

Fig. 7. A medical image batch transmission system

\subsection{A prototype transmission system}

Fig. 7 shows a prototype system for the multi-transmission optimization processing of the medical images. Fig. 7(a) is an example of the backend interface of offline medical image processing. The two MUAs in this figure have been identified by using two blue polygon lines. Fig. 7(b) demonstrates one of the receiver clients interface in which the IBs have been reconstructed and displayed. Comparing the two figures, it is obvious that: 1) the pixel resolutions of the MUAs in Fig. 7(b) keep original; 2) the pixel resolution of the non-MUA part in Fig. 7(b) is significantly lower than that of in Fig. 7(a). 


\subsection{Effectiveness of the MTO method}

To objectively evaluate the effectiveness of the MTO method and compare the image qualities after the image distortion processing, in the first experiment, a metric called the peak signal to noise ratio (PSNR)[18] is adopted to measure the quality of reconstruction of the image compression. The signal in this case is the original data, and the noise is the error introduced by the MTO compression. The PSNR is an approximation to human perception of reconstruction quality, represented as follows:

$$
P S N R=10 \log _{10} \frac{\left(2^{B}-1\right)^{2}}{M S E}
$$

where MSE refers to mean squared error, denoted as: $M S E=\frac{1}{m n} \sum_{i=0}^{m-1} \sum_{j=0}^{n-1}[I(i, j)-K(i, j)]^{2} ; B$ is the image bits.

\subsubsection{Effect of $\delta$ on PSNR}

This experiment studies the effect of $\delta$ on the quality of image reconstruction. Fig. 8 illustrates an example of the original image and the reconstruction of the same image based on different $\delta$ and different number of clusters(CNo), respectively. The pixel resolution of the RIB is fixed that is $80 \mathrm{dpi}$. It seems that the image reconstruction quality is relatively poor when the granularity $\delta$ is smaller. This is because it is hard to find the correct RIBs to accurately capture the visual similarity of the other $I B s$ in the corresponding cluster when the number of the $I B s$ is getting small, especially in Figs. 8(b)(c). Meanwhile, when $\delta$ becomes larger, the image quality is getting better since it is easy to find the corresponding RIBs in the related NIBs which can be effectively represented by them. It is worth mentioning that although a minor image distortion occurred after the image reconstruction processing, this will not affect the examining and diagnosis since the image qualities of the MUAs are still kept original. Therefore, there is a tradeoff among the image quality, the granularity $(\Delta)$ for resolutions and the granularity $(\delta)$ for the size of image blocking.

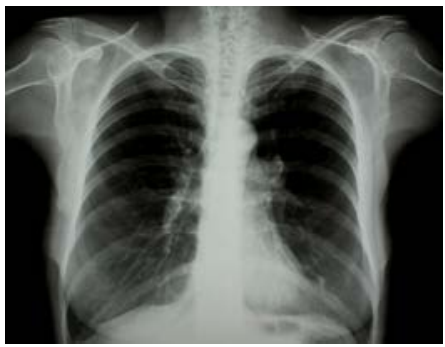

(a). Before

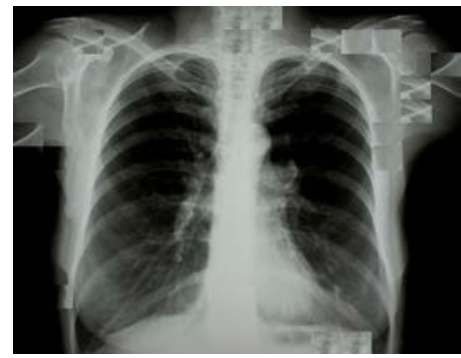

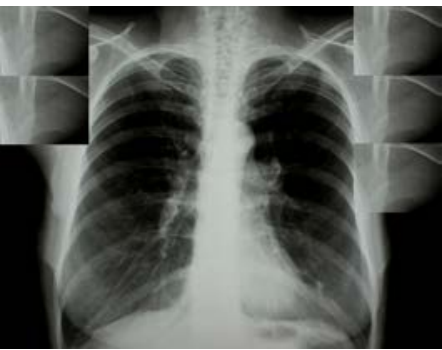

(b). $\delta=5 \times 5, \mathrm{CNo}=6$

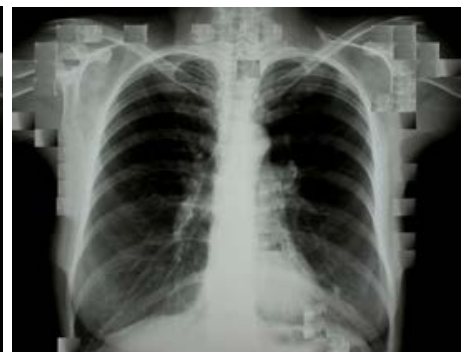

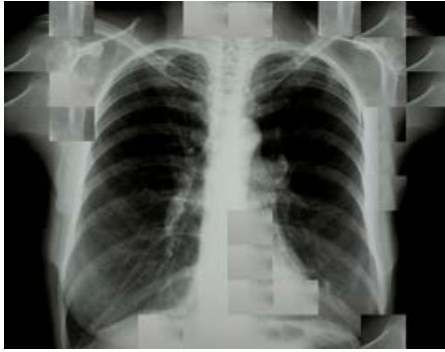

(c). $\delta=10 \times 10, \mathrm{CNo}=8$

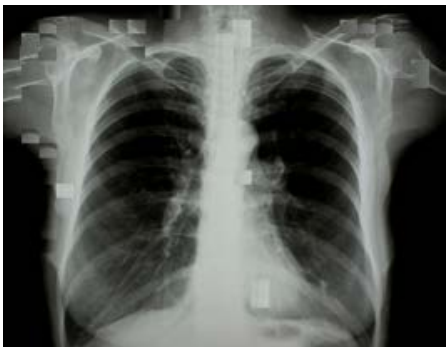



(d). $\delta=15 \times 15, \mathrm{CNo}=9$
(e). $\delta=20 \times 20, \mathrm{CNo}=11$
(f). $\delta=25 \times 25, \mathrm{CNo}=15$

Fig. 8. Effect of $\delta$ (RIB.dpi=80 dpi)

Furthermore, the two types of image sets (i.e., real images and synthetic ones) are used to objectively evaluate the effect of $\delta$ on the image reconstruction qualities by using the PSNR. In Fig. 9, with the increase of $\delta$, the PSNR values are first increasing gradually for both of the two image sets. When $\delta$ is larger than $20 \times 20$, it seems that their corresponding PSNR values can not increase anymore. This is because when $\delta$ is smaller, it is hard to reconstruct the original image perfectly since the suitable RIBs are hard to be obtained due to the small number of the IBs. On the contrary, when $\delta$ increases, as more IBs are partitioned, so it is relatively easy to find a suitable RIB to represent all IBs in the corresponding cluster.

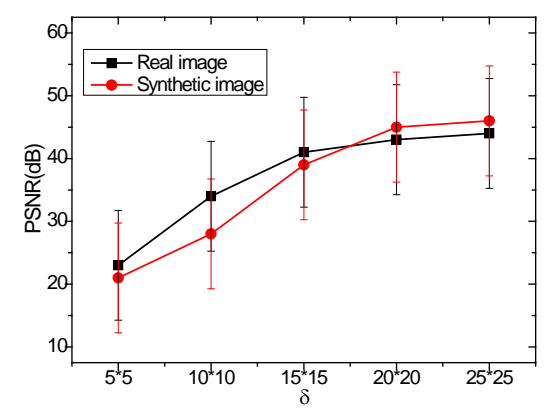

Fig. 9. Effect of $\delta$ on $\operatorname{PSNR}(\Delta=20)$

\subsubsection{Effect of $\triangle$ on PSNR}

Next, this subsection evaluates the effect of $\Delta$ on the image reconstruction quality in which $\delta=20 \times 20$ as an optimal one. Similar to Section 4.2.1, Fig. 10 shows an example of the original image and the reconstruction of the same image when $\delta=$ $20 \times 20$ and $\Delta=5$. Suppose that the lower and upper bounds of $d p i$ are 40, 200, respectively. Comparing with Figs. 10(b) and 10(c), it seems that the resolution of the non-MUA part in image in Fig. 10(b) is much lower than that of in Fig. 10(c). When the resolutions of the RIBs are larger than $80 \mathrm{dpi}$, the image reconstruction qualities are approximately similar.

In this experiment, two types of image sets are used mentioned above. Fig. 11 shows the effect of RIB.dpi on PSNR with different $\Delta$, where $\delta$ is fixed (e.g., 20×20). From Figs. 11(a-d), it is interesting to see that with the increase of RIB.dpi, the PSNR values are first increasing gradually for both of the two image sets. When RIB.dpi is larger than 80(Fig. 11(a)), 100(Fig. 11(b)), 110(Fig. 11(c)), and 125(Fig. 11(d)), respectively, the variance ranges of their corresponding PSNR values become smaller and the PSNR values can not increase anymore. 


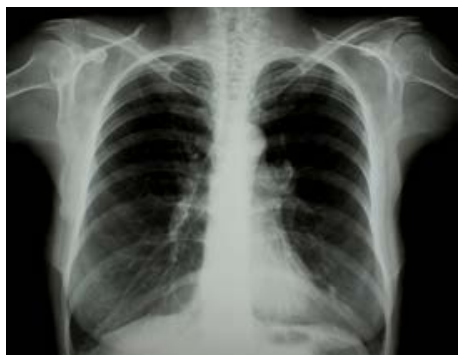

(a). Before

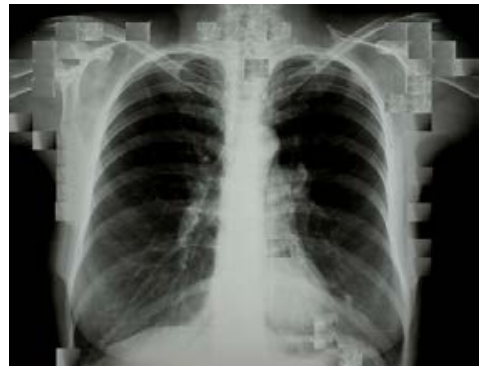

(d). RIB.dpi=120dpi

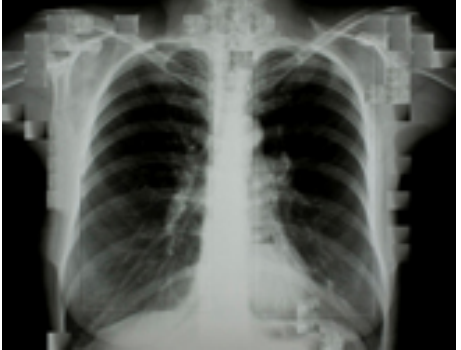

(b). $R I B \cdot d p i=40 \mathrm{dpi}$

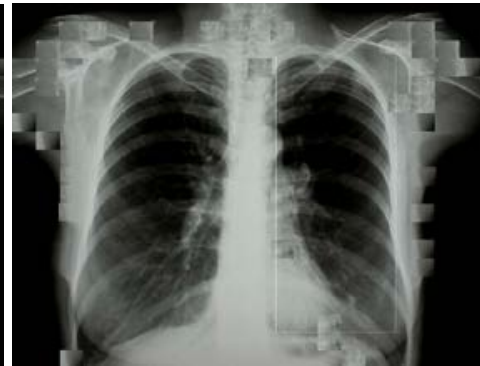

(e). RIB.dpi=160dpi

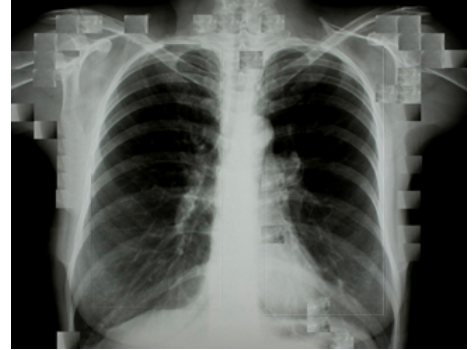

(c). RIB.dpi=80dpi

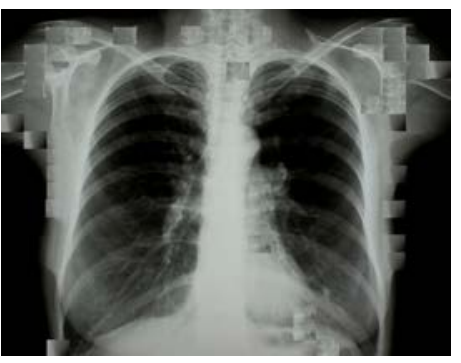

(f). $R I B \cdot d p i=200 \mathrm{dpi}$

Fig. 10. An example of image reconstruction $(\Delta=5$ and $\delta=20 \times 20)$

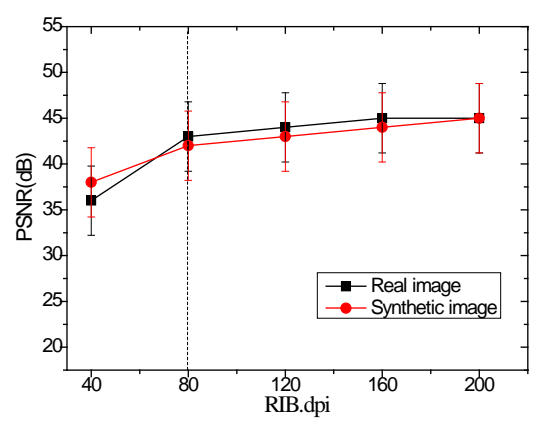

(a). $\Delta=5$

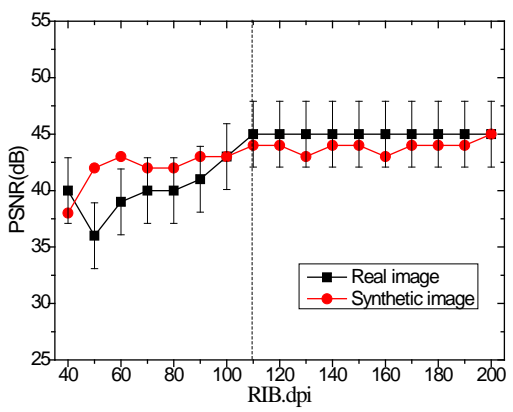

(c). $\Delta=17$

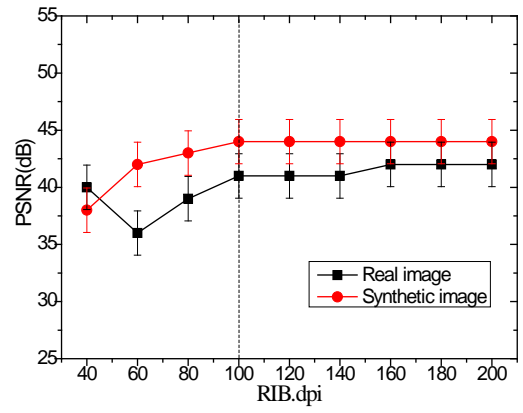

(b). $\Delta=9$

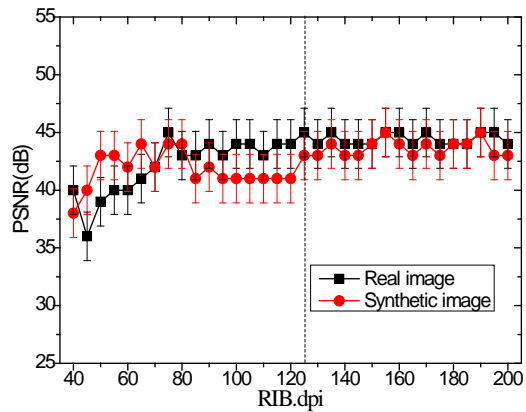

(d). $\Delta=33$

Fig. 11. Effect of RIB.dpi on PSNR with different $\Delta$

\subsection{Effect of number of transmission images}

This experiment studies the effect of the transmission images on the performance of the MTO processing by using the above two kinds of image data. The average data sizes for the real and synthetic images are $500 \mathrm{kB}$ and 10MB, respectively. Before the experiment, a definition of a speedup is obtained: 


$$
\text { Speedup }=\frac{\text { Time for the } m \text { image transmissions }}{\text { Time for the MTO }}
$$

Method 1 uses the traditional transmission scheme and method 2 adopts the MTO one. As illustrated in Fig. 12, when the bandwidth is relatively stable, the total transmission time using the MTO is superior to that of the traditional one. Meanwhile, with the increase of the image data size (i.e., the number of transmission images), the speedups are significantly larger than one and increase gradually. Additionally, for the synthetic data, the performance gap of the two approaches becomes enlarged when the image size is increasing. The reason is that our proposed hybrid pixel resolution approach can effectively reduce the transmitted image data, especially for the large ones.

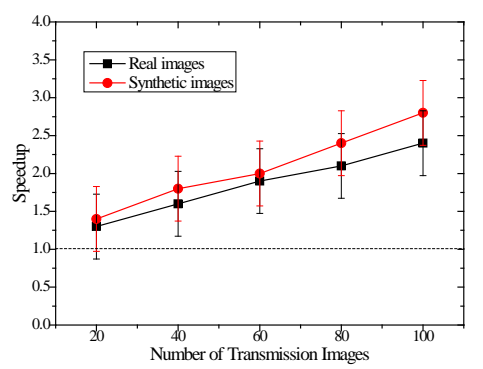

Fig. 12. Effect of number of transmission images

\subsection{Effect of image size}

This experiment empirically compares the effect of the image size on the performance of the MTO processing by using the above two kinds of image data. Method 1 uses traditional transmission method and method 2 adopts the MTO method. The horizontal axis means the total data size of the transmission images. The number of the images is 10 . As illustrated in Fig.13, when the bandwidth is relatively stable, the total transmission time using the MTO is superior to that of the traditional one. Meanwhile, with the increase of the image data size, the performance gap becomes larger. This is because the data size of the images to be transmitted is increasing so rapidly that the images cannot be sent to the destination nodes quickly. Further, for the synthetic data, the performance gap of the two approaches becomes enlarged when the image size increases. The reason is that our proposed hybrid pixel resolution approach can effectively reduce the transmitted image data, especially for large images.

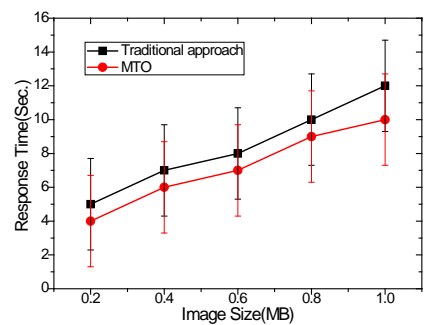

(a). Real dataset

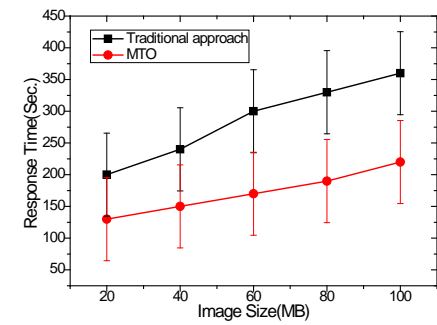

(b). Synthetic dataset

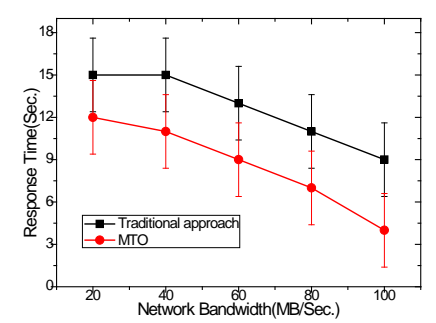

(a). Real dataset

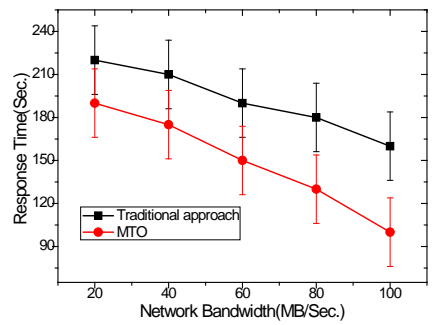

(b). Synthetic dataset

Fig. 13. Effect of image size

Fig. 14. Effect of network bandwidth 


\subsection{Effect of network bandwidth}

This experiment investigates the effect of the network bandwidth on the performance of the MTO processing by using the two data sets. For the real and synthetic datasets, the average data sizes of the two kinds of images are $500 \mathrm{~KB}$ and $50 \mathrm{MB}$, respectively. Method 1 uses traditional transmission method and method 2 adopts the MTO. Fig. 14 shows when the image data sizes are fixed, the total response time using the method 2 is superior to that of method 1 . Meanwhile, with increasing bandwidth, the response time decreases gradually and the performance gap becomes larger especially for the large synthetic image data. This is because in the MTO, compared with the original image data size, it has been much reduced based on the network bandwidth, the image content, and so on, causing the transmission cost to decrease accordingly.

\subsection{Effect of $\Delta$}

This experiment proceeds to test the effect of $\Delta$ on the transmission and storage costs, respectively, by using the two datasets. The data sizes in the two image sets are same as Section 4.3. In Fig. 15, when $\Delta$ ranges from 3(5) to 15(25), the transmission cost gradually decrease; while in Fig. 16, the storage cost increases with the increase of $\Delta$. This is because when $\Delta$ is small, the data size of each $I B$ replica becomes relatively large. Thus, the transmission cost becomes higher. Similarly, the total number of the $I B$ replicas increases when $\Delta$ increases, which leads to the larger storage cost. So, to obtain a tradeoff between transmission cost and storage cost, an optimal $\Delta$ is critically important to the MTO processing.

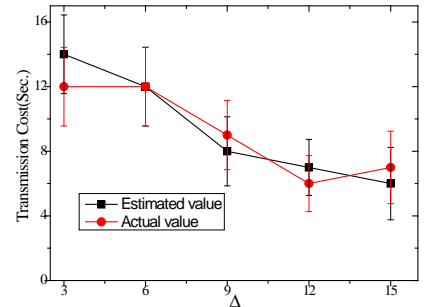

(a). Real dataset

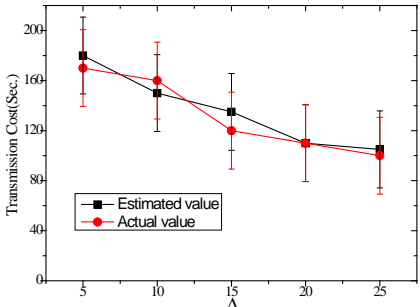

(b). Synthetic dataset

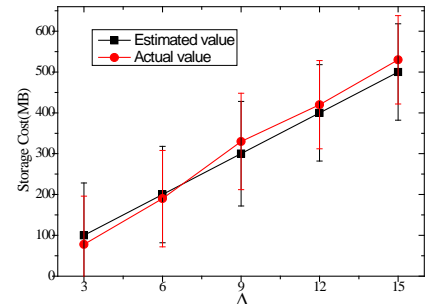

(a). Real dataset

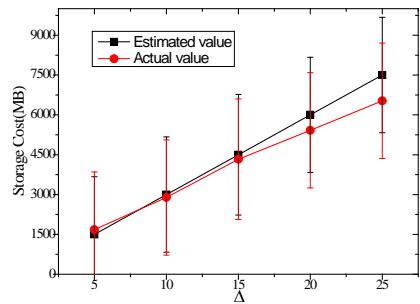

(b). Synthetic dataset

Fig. 15. Effect of $\Delta$ on transmission cost

Fig. 16. Effect of $\Delta$ on storage cost

\subsection{Comparison of transmission efficiency and robustness}

This experiment compares the transmission efficiency and robustness of the two transmission schemes: 1) our proposed MTO method and 2) the progressive image transmission (PIT) [18] by using the synthetic dataset. In Fig. 17(a), when the image data size is increased from 20MB to $100 \mathrm{MB}$, the transmission time of the MTO method gradually increases but better than that of the PIT one. This is because compared with the mixed pixel resolution-based image reconstruction of the MTO scheme, the PIT adopts original resolution to reconstruct the images. The data size to be transferred by the PIT is larger than that of the MTO.

To evaluate the effect of image size on the transmission robustness, the synthetic image dataset can be used in which the 
images have been divided into five groups in terms of the data size such as $5 \mathrm{MB}, 10 \mathrm{MB}, 20 \mathrm{MB}, 50 \mathrm{MB}$, and $100 \mathrm{MB}$. The transmission robustness $(T R)$ is defined below.

$$
T R=\frac{\text { Number of successful data transmissions }}{\text { Total number of data transmissions }}
$$

As shown in Fig. 17(b), even with the increase of data size, the successful data transmission ratio (i.e., TR) remains extremely close to $100 \%$ by using the image blocking technique. For the data transmission without adopting the MTO method, the average $T R$ is decreased to $87 \%$ at the data size of $20 \mathrm{MB}$ and approaches to zero when the data size is larger than 50MB, since it is hard to transfer a complete large image successfully. Based on the experimental result, to guarantee a high successful data transmission ratio, it is necessary to transfer a large image only through the image blocking method in a limited network bandwidth.

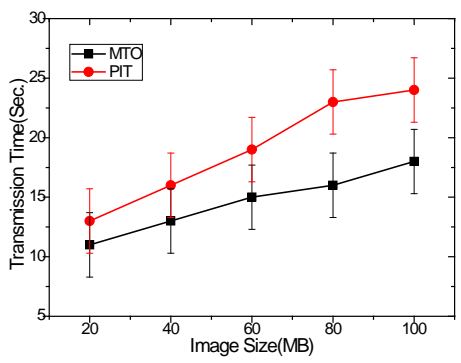

(a). Image size vs. transmission efficiency

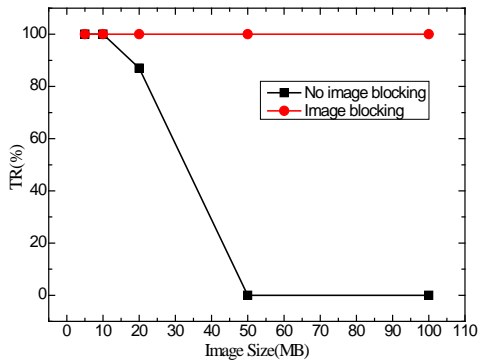

(b). Image size vs. transmission robustness

Fig. 17. Effect of the MTO on TR

\section{Conclusion}

The paper presented a multiple transmission optimization method for large medical images called the MTO in resourceconstraint mobile telemedicine systems. The proposed MTO is specifically designed for the batch transmission of the multiple large medical images concurrently under low and unstable network bandwidth. Two enabling techniques, namely, NIB grouping scheme, and adaptive RIB replicas selection are proposed to reduce the data communication cost. The experimental studies demonstrate that the proposed MTO method is more suitable for the multiple medical image transmission in minimizing the network communication cost and maximizing the parallelism in I/O and CPU.

\section{Acknowledgement}

The authors would like to thank the editors and anonymous reviewers for their helpful comments. This work is partially supported by the Program of National Natural Science Foundation of China under grant No. 61272188, 61540064; the Ministry of Education of Humanities and Social Sciences Project under grant No. 14YJCZH235; the “Qianjiang Talent” Project of Zhejiang Province under grant No. QJD1402017. 


\section{References}

[1] A. Qureshi, A. Shoeb, J. Guttag. Building a high-quality mobile telemedicine system using network striping over dissimilar wireless wide area networks In Int'l Conf. of the Engineering in Medicine and Biology Society. 3942-3945. 2005.

[2] H. Muller, N. Michoux, D. Bandon, et al. A review of content-based image retrieval systems in medical applicationsclinical benefits and future directions. Int'l Journal of Medical Informatics. 2004. 73:1-23.

[3] Y. Zhuang, N. Jiang, Z-A. Wu, Q. Li, etc. Efficient and Robust Large Medical Image Retrieval in Mobile Cloud Computing Environment. Information Sciences. Vol.263. 60-86. 2014.

[4] Tzou, K. H. Progressive image transmission: a review and comparison of techniques, Optical Engineering. 1987.(26), 581-589.

[5] C. J. Turner, L. L. Peterson. Image transfer: an end-to-end design. In ACM SIGCOMM. 1992. 258-268.

[6] John, M. D., Georey, M. D., Song, X.Y. Fast lossy internet image transmission. In ACM Multimedia. 1995.

[7] Raman, S., Balakrishnan, H., Srinivasan, M. An image transport protocol for the Internet. In ICNP. 2000. 209-219.

[8] Allcocka, B., Bestera, J., Bresnahan, J., etc. Data management and transfer in high-performance computational grid environments. In Parallel Computing. 2002. 28(5), 749-771.

[9] Aziz, S.M., D. M. Pham. Energy Efficient Image Transmission in Wireless Multimedia Sensor Networks. In IEEE Communications Letters, Vol.17, Issue: 6, June 2013. pp. 1084 - 1087.

[10] J.H. Kim, and W.J. Song. Pyramid-structured progressive image transmission using quantization error delivery in transform domains, IEE Vision, Image and Signal Processing. 1996. (143),132-136.

[11] C. C. Chang, F. C. Shine, and T.S. Chen. A new scheme of progressive image transmission based on bit-plane method, In Asia-Pacific Conference on Communications and Fourth Optoelectronics and Communications Conference, 1999. 2, 892895.

[12] Hashimoto, M., Koike, A., Matsumoto, S. Hierarchical image transmission system for telemedicine using segmented wavelet transform and Golomb-Rice codes. In Global Telecommunications Conference(GLOBECOM '99), Vol.4, 1999. pp: 2208 - 2212.

[13] V.G. Ruiz, J.J. Fernández, and I. García. Image compression for progressive transmission. In the Nineteenth IASTED Int'l Conf. on Applied Informatics: Advances in Computer Applications. 2001. 519-524, Austria.

[14] C. C. Chang, T.K. Shih, and I.C. Lin. Guessing by neighbors: an efficient reconstruction method for transmitting image progressively, In The Visual Computer. 2003. (19)5, 342-353.

[15] C. C. Chang, and M.N. Wu. A color image progressive transmission method by common bit map block truncation coding approach, In Int'l Conf. on Communication Technology. 2003. (2), 1774-1778.

[16] T. Lin, P. Hao. Compound image compression for real-time computer screen image transmission. In IEEE TIP. 2005. 14 (8): 993-1005.

[17] Y. Sun, Z-X. Xiong, Progressive Image Transmission over Space-Time Coded OFDM-Based MIMO Systems with Adaptive Modulation. In IEEE TMC. 5(8), August 2006. pp. 1016-1028

[18] R.C. Chang, T.K. Shih, H.H. Hsu. A Strategic Decomposition for Adaptive Image Transmission. In Journal of Information Science and Engineering. 2008. 24(3): 691-707.

[19] D.H. Gao, D.H. Liu, Y.Q. Feng, et al. A Robust Image Transmission Scheme for Wireless Channels Based on Compressive Sensing. Advanced Intelligent Computing Theories and Applications. With Aspects of Artificial Intelligence. 2010. 6216, 334- 341.

[20] P. S. Boluk, S. Baydere, A. E. Harmanci. Robust Image Transmission over Wireless Sensor Networks. Journal Mobile Networks and Applications. 16(2), April 2011. pp. 149- 170.

[21] S.S. Arslan, P.C.Cosman, L.B.Milstein, Generalized unequal error protection LT Codes for progressive data transmission, In IEEE TIP, 21(8), pp. 3586-3597, 2012. 
[22] H-S. Xua, K. Hua, H-G. Wang. Adaptive FEC coding and cooperative relayed wireless image transmission. Digital Communications and Networks. Vol. 1, Issue 3, August 2015, pp. 213-221

[23] Maani. R, Camorlinga S., Amason N. A parallel method to improve medical image transmission. Journal of Digit Imaging. 2012. 25(1).

[24] R. Hemalatha, S. Radha, S.Sudharsan. Energy-efficient image transmission in wireless multimedia sensor networks using block-based Compressive Sensing. Journal of Computers and Electrical Engineering. Vol. 44 Issue C, May 2015, pp. 67-79.

[25] S. Manimurugan, C. Narmatha. Secure and Efficient Medical Image Transmission by New Tailored Visual Cryptography Scheme with LS Compressions. Int’l Journal of Digital Crime and Forensics archive. Vol. 7 Issue 1, January 2015. pp. 2650.

[26] Ali Ai-Hai, Gheith Abandah, Noor Hussein. Crypto-based algorithms for secure medical image transmission. IET Information Security. Vol. 9, Issue 6. pp. 365-373. 2015.

[27] Gal, V., Kerre, E., Tikk, D. Organ Detection in Medical Images with Discriminately Trained Deformable Part Model. In IEEE $9^{\text {th }}$ Int'l Conf. on Computational Cybernetics (ICCC). pp. 153- 157. 2013.

[28] Y. Zhuang, N. Jiang, Q. Li, Dickson K.W. Chiu, H. Hu. Personalized and Efficient Social Image Transmission Scheme in Mobile Wireless Network. Multimedia Tools and Applications. 75(6). 2931-2968, 2016.

[29] B.J. Frey, D. Dueck, Clustering by passing messages between data points, Science 5814 (315) (2007) $972-976$.

[30] The Android platform, www.google.com/android, 2010

[31] MySQL. http://www.mysql.com/. 2011

[32] The medical image archive. http://www.ece.ncsu.edu/imaging/Archives/ImageDataBase/Medical/index.html. 2010. 